\title{
Angular Vectors in the Theory of Vectors
}

\author{
Yevhen Kharchenko $^{1}$ \\ ${ }^{1}$ Zaporizhzhia, Ukraine \\ Correspondence: Yevhen Kharchenko, 14 October 9-174, Zaporizhzhia, Ukraine, 69123. E-mail: Ujin-X@meta.ua
}

Received: June 16, 2017 Accepted: July 28, 2017 Online Published: September 12, 2017

doi:10.5539/jmr.v9n5p71 URL: https://doi.org/10.5539/jmr.v9n5p71

\begin{abstract}
The theory of angular vectors, which allows modelling of the properties of angular physical quantities, is considered. The meaning of the cross product of vectors was radically revised and changed. Formulas for finding torque and angular velocity in a coordinate-vector form with a correct mapping of their directions were deduced. Described definition of the inverse vector and its properties. The inversed vector allows us to perform vector cross division operations.
\end{abstract}

Keywords: angular vector, cross product of vectors, inverse vector, theory of vectors

\section{Introduction}

For describing the physical quantities with a direction (velocity, acceleration, force, etc.) the vector algebra theory successfully utilize rectilinear vectors (Dennis \& Michael, 2006).

But there are also physical quantities with an angular direction (angular velocity, angular acceleration, torque, moment of inertia, etc.). These quantities are also described mathematically as rectilinear.

The problem is that when we try to present angular quantities as rectilinear, we get a discrepancy between the directions of the physical quantity and the mathematical vector. We obtain a mathematical vector (Kumar, 2008) directed perpendicular to the direction of action of the physical quantity. And the physical quantity is directed, as it may be, around the circle in the plane of its action. For example, Fig. 1 shows the rotating body, and the difference in the actual direction of the angular velocity of the body and the mathematical angular velocity vector.

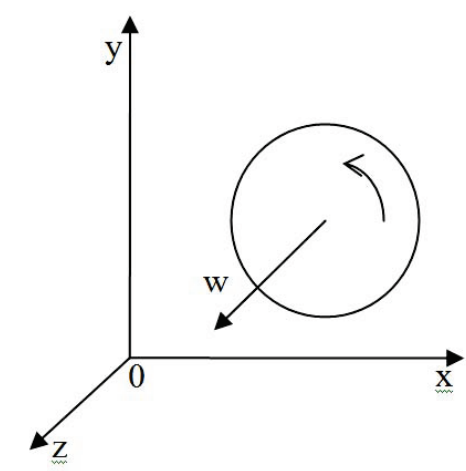

Fig. 1. Graphical representation of the mathematical angular velocity vector and the direction of the physical angular velocity as the body rotates

Let us consider in more detail the example of a problem with inconsistent directions.

Let us find the magnitude and direction of the torque on the propeller shaft, when birds are sitting on its blades, Fig. 2. The propeller is in the $0 \mathrm{XY}$ plane.

When solving single human-aided problems, equations of the general form are used (Boothroyd \& Poli, 1980). In these equations, at any change in the arrangement of forces, we must manually indicate the directions of the torque generated, in spite of the fact that the forces and the distance can be represented in a coordinate form.

$$
M=+r 1 \cdot F 1-r 2 \cdot F 2
$$

For calculation in automatic programs, the formulas for finding of the moment in coordinate-vector form are used (Szolga, 


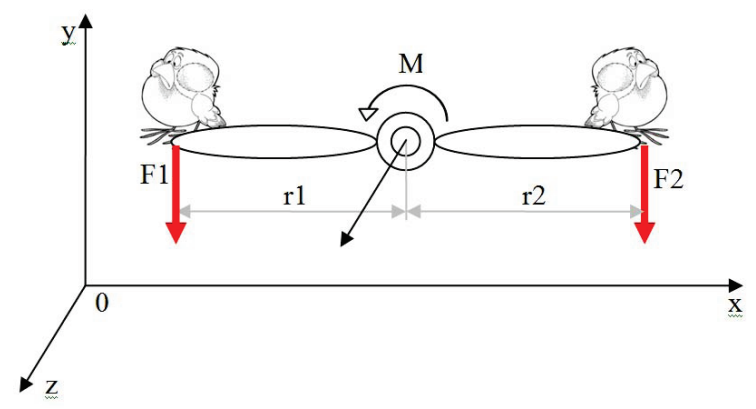

Fig. 2. Presence of forces and torque on the propeller

2010). But there is also confusion with the directions of the torque in them:

$$
\begin{aligned}
& \bar{M}=\bar{M}_{1}+\bar{M}_{2} \\
& \bar{M}_{1,2}=\bar{r} \times \bar{F}=\left(r_{y} F_{z}-r_{z} F_{y}\right) \bar{i}+\left(r_{z} F_{x}-r_{x} F_{z}\right) \bar{j}+\left(r_{x} F_{y}-r_{y} F_{x}\right) \bar{k}=M_{x} \bar{i}+M_{y} \bar{j}+M_{z} \bar{k} \\
& \bar{M}_{1,2}=0 \bar{i}+0 \bar{j}+\left(r_{x} F_{y}-r_{y} F_{x}\right) \bar{k}=M_{z} \bar{k}
\end{aligned}
$$

We do not have confusion with signs of forces $(\mathrm{F} 1, \mathrm{~F} 2)$ as in general formulas, but the direction of the general mathematical moment $\bar{M}$ and the actual physical moment do not coincide. Because the total moment is obtained as a rectilinear vector directed parallel to the $\mathrm{OZ}$ axis for the given problem. As a result, we can not unequivocally indicate in which direction the physical torque is directed, clockwise, or counterclockwise.

In other words we cannot correctly display the direction of angular physical quantities in the coordinate system.

This paper shows how you can change the description of a mathematical model of angular quantities and consider them separately from rectilinear. Described theory of angular vectors is in good agreement with the theory of rectilinear vectors and it is its complement. At that the possibilities of application of the general theory of vector modelling expand.

To make a summary, angular vectors in the used formulas are almost indistinguishable from rectilinear vectors. Their main difference is the presentation of angular vectors and their projections in the coordinate system. The same changes in cross product of vectors are proposed. And thanks to the described inversed vectors we receive ability to perform vector division.

\section{Theory of Angular Vectors}

To describe physical quantities with magnitude and direction, the mathematical model is applied which is called the vector. Physical quantities vary in type (speed, acceleration ...), so you need to remember that vectors are also conditionally divided according to the type of physical quantities represented.

\subsection{Designation of Vectors}

Angular vector has magnitude (quantity) and direction, but has no exact location, and it is located in the plane of action of displayed physical quantity. This property of all angular vectors can be shown on the examples for kinematic and power quantities.

Example 1: Satellite of differential mechanism has a complex movement and rotates around two axes: the primary axis and the moveable axis. Therefore, it has three angular velocities: around the main axis, around the mobile axis and the common angular velocity, which does not have its own axis. But all these angular velocities have a direction and a magnitude.

Even for the angular velocity vector, which has its axis of rotation, we cannot specify the location on the plane, where it is located at any given time.

Example 2: Angular vector of torque acquires the exact location only if there are relations in which the torque generates the forces. Location of relations can be changed, therefore force change, and the magnitude and direction of the moment does not change.

\section{Geometric Designation}

Methods of displaying of angular vectors are known for a long time. It can be curved arrows displaying kinematic quantities Fig.3 (angular velocity, angular acceleration). Or arrows (two arrows) with a lever that display power quantities (torque, moment of inertia). These designations conditionally display the presence of angular vector in the plane. 

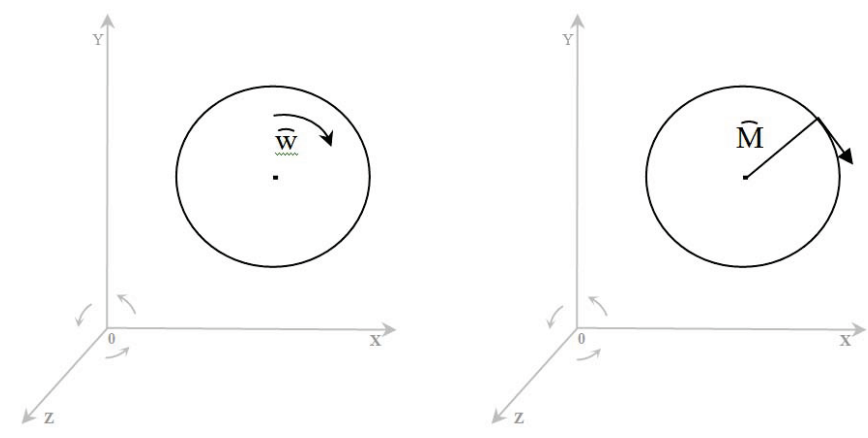

Fig. 3. Designation of angular vectors

\section{Mathematical designation}

To differ from the rectilinear vectors angular vector can be designated with the curved arrow (line) above the letter $\widehat{b}$.

The angular vector is denoted in coordinate system as a projection on the coordinate plane.

$$
\widehat{b}\left(b_{x y}, b_{y z}, b_{z x}\right)
$$

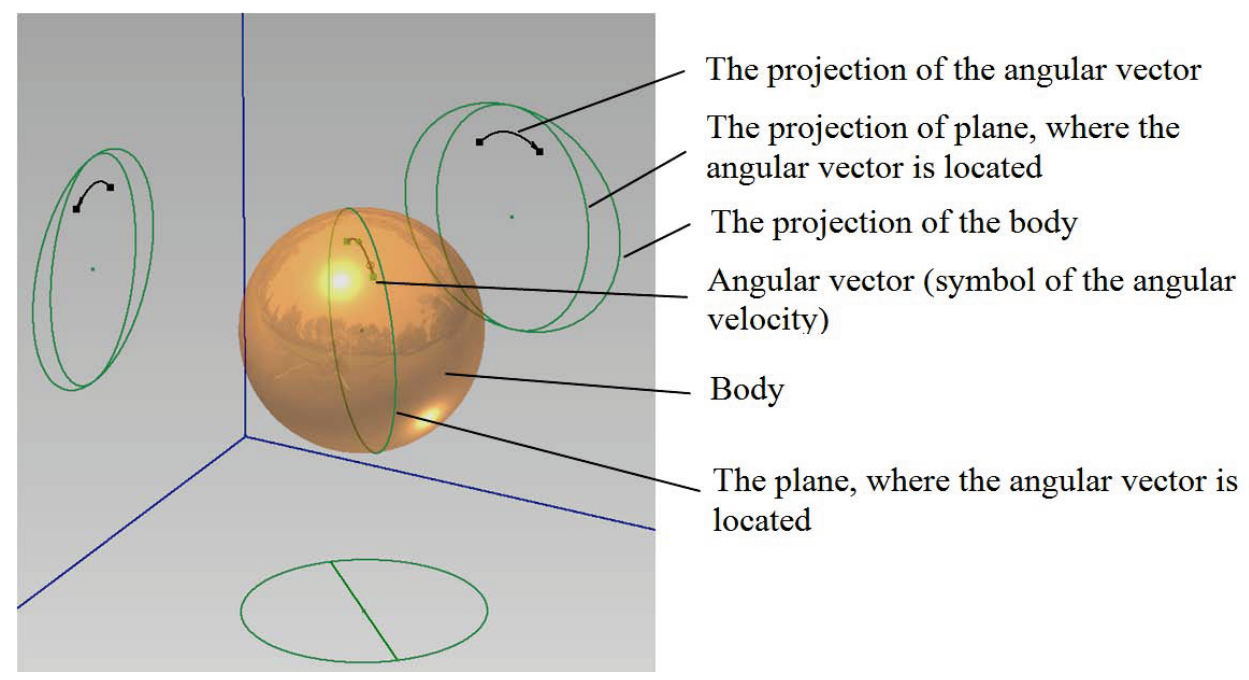

Fig. 4. Designation of angular vector in the coordinate system

This form of recording (implicit) shows only the direction and magnitude of the vector, and does not define it precisely in the coordinate system. This vector can be one of the many parallel, equally directed angular vectors.

It is the only way to designate the physical quantity without the exact location that lies on any plane.

Angular vector cannot be projected to the coordinate axes, so as you transfer to the coordinate axes you lose direction of the angular vector. The projection of the angular vector onto the plane, which is perpendicular to the plane in which it is located is equal to zero.

Angular vector can be represented graphically, as the area of any shape (for the torque - the area of a parallelogram).

In the coordinate system in addition to linear positive direction (coordinate axis), one shall additionally indicate the angular positive directions, Fig.5. For example, in the form of curved arrows in each plane. In describing, the positive angular direction is indicated from one coordinate axis to another. For example, for Fig.5(b), the positive angular direction between the axes is directed $O x \rightarrow O y \rightarrow O z$.

Introduction of angular direction of the coordinate system allows you to specify positive direction of the projections of angular vectors in coordinate planes.

When designating the projections of angular direction on the XY plane, the order of the indices shall indicate their angular 


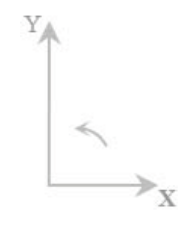

a)

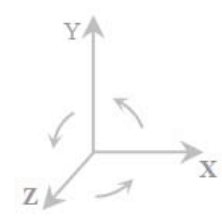

b)

Fig. 5. Determination of angular direction in the coordinate system

direction. Thus, for the projection of the vector $b_{x y}$ the positive direction in the coordinate axes will be $O x \rightarrow O y$. Then

$$
b_{x y}=-b_{y x} \quad b_{y z}=-b_{z y} \quad b_{z x}=-b_{x z}
$$

Angular vector magnitude is calculated the same as for linear vector.

$$
|\widehat{b}|=\sqrt{b_{x y}^{2}+b_{y z}^{2}+b_{z x}^{2}}
$$

Angular unit vectors (basis vectors), as well as for rectilinear basis vectors reflect the positive direction in the chosen coordinate system, Fig. 6.

In this paper we shall conventionally take the names of angular basis vectors which belong to the planes: $\hat{l} \in O X Y($ plane $)$, $\widehat{m} \in O Y Z, \widehat{n} \in O Z X$

Rectilinear basis vectors $\bar{i}, \bar{j}, \bar{k} \quad$ - specify the scale of coordinate system and characterized its type.

Angular basis vectors $\hat{l}, \widehat{m}, \widehat{n} \quad$ - show only positive angular direction in coordinate planes, their size depends on the size of the rectilinear basis vectors (in details in cross product of vectors).

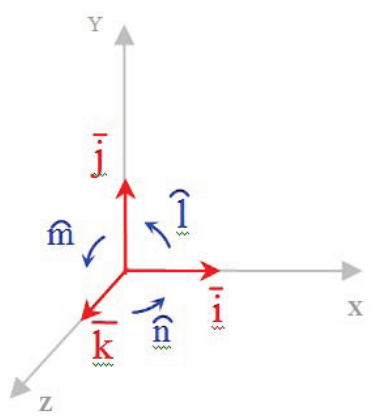

Fig. 6. Angular basis vectors in a coordinate system

Let's consider the basic mathematical operations on angular vectors:

\subsection{Addition of Vectors}

This operation is possible only among similar vectors. It's the same for both rectilinear and angular vectors. The only difference is that addition of angular vectors cannot be represented graphically because angular vectors look like squares of any shapes.

The sum of the vectors is equal to the sum of their projections:

$$
\widehat{a}+\widehat{b}=\widehat{c} \quad \widehat{a}+\widehat{b}=\left(a_{x y}+b_{x y}, a_{y z}+b_{y z}, a_{z x}+b_{z x}\right)
$$

All vectors subject to the associativity property $(\widehat{a}+\widehat{b})+\widehat{c}=\widehat{a}+(\widehat{b}+\widehat{c})$

All vectors subject to the associativity commutativity $\widehat{a}+\widehat{b}=\widehat{b}+\widehat{a}$ 
For any vector $\widehat{a}$ opposite vector $-\widehat{a}$ exists such that $\widehat{a}+(-\widehat{a})=0$

Particular cases of addition of angular vectors:

Angular vector can be represented as the sum of the vectors that are in planes of coordinate systems

$$
\widehat{b}\left(b_{x y}, b_{y z}, b_{z x}\right)=\widehat{c}\left(b_{x y}, 0,0\right)+\widehat{d}\left(0, b_{y z}, 0\right)+\widehat{t}\left(0,0, b_{z x}\right)
$$

Angular vector can be represented as the sum of the vectors each of which is perpendicular to one of the coordinate planes. At that the original vector is twice more than the received vectors.

$$
\begin{gathered}
2 \widehat{b}\left(2 b_{x y}, 2 b_{y z}, 2 b_{z x}\right)=\widehat{c}\left(b_{x y}, b_{y z}, 0\right)+\widehat{d}\left(0, b_{y z}, b_{z x}\right)+\widehat{t}\left(b_{x y}, 0, b_{z x}\right) \\
2 \widehat{b}\left(2 b_{x y}, 2 b_{y z}, 2 b_{z x}\right)=\widehat{c}\left(2 b_{x y}, b_{y z}, 0\right)+\widehat{d}\left(0, b_{y z}, 2 b_{z x}\right) \\
2 \widehat{b}\left(2 b_{x y}, 2 b_{y z}, 2 b_{z x}\right)=\widehat{d}\left(0,2 b_{y z}, b_{z x}\right)+\widehat{t}\left(2 b_{x y}, 0, b_{z x}\right) \\
2 \widehat{b}\left(2 b_{x y}, 2 b_{y z}, 2 b_{z x}\right)=\widehat{c}\left(b_{x y}, 2 b_{y z}, 0\right)+\widehat{t}\left(b_{x y}, 0,2 b_{z x}\right)
\end{gathered}
$$

\subsection{Angles between the Plane of Angular Vector and the Planes of Coordinate Systems}

Angular vector forms with its coordinate projections a angles $\varphi_{1}, \varphi_{2}, \varphi_{3}$ :

$$
\left\{\begin{array}{l}
b_{x y}=|\widehat{b}| \cdot \cos \left(\varphi_{1}\right) \\
b_{y z}=|\widehat{b}| \cdot \cos \left(\varphi_{2}\right) \\
b_{z x}=|\widehat{b}| \cdot \cos \left(\varphi_{3}\right)
\end{array}\right.
$$

$$
\left\{\begin{array}{l}
\varphi_{1}=\arccos \left(\frac{b_{x y}}{\sqrt{b_{x y}^{2}+b_{y z}^{2}+b_{z x}^{2}}}\right) \\
\varphi_{2}=\arccos \left(\frac{b_{y z}}{\sqrt{b_{x y}^{2}+b_{y z}^{2}+b_{z x}^{2}}}\right) \\
\varphi_{3}=\arccos \left(\frac{b_{z x}}{\sqrt{b_{x y}^{2}+b_{y z}^{2}+b_{z x}^{2}}}\right)
\end{array}\right.
$$

\subsection{Inverse Vector}

Inverse vector $\bar{a}^{\prime}$ is a vector which is co-directed with a vector $\bar{a}$ and differs from it in size, fig. 7 .

$$
\left|\overline{a^{\prime}}\right|=\frac{1}{|\bar{a}|}
$$

Projections on the axis of inverse rectilinear vector are equal:

$$
a_{x}^{\prime}=a_{x} \frac{\left|\bar{a}^{\prime}\right|}{|\bar{a}|}=\frac{a_{x}}{|\bar{a}|^{2}}=\frac{a_{x}}{\left(\sqrt{a_{x}^{2}+a_{y}^{2}+a_{z}^{2}}\right)^{2}}=\frac{a_{x}}{a_{x}^{2}+a_{y}^{2}+a_{z}^{2}}, \quad a_{y}^{\prime}=\frac{a_{y}}{a_{x}^{2}+a_{y}^{2}+a_{z}^{2}}, \quad a_{z}^{\prime}=\frac{a_{z}}{a_{x}^{2}+a_{y}^{2}+a_{z}^{2}}
$$

These connections are also correct for the inverse angular vector:

$$
\begin{gathered}
\mid \widehat{a^{\prime} \mid=\frac{1}{|\widehat{a}|}} \\
a_{x y}^{\prime}=\frac{a_{x y}}{a_{x y}^{2}+a_{y z}^{2}+a_{z x}^{2}}, \quad a_{y z}^{\prime}=\frac{a_{y z}}{a_{x y}^{2}+a_{y z}^{2}+a_{z x}^{2}}, \quad a_{z x}^{\prime}=\frac{a_{z x}}{a_{x y}^{2}+a_{y z}^{2}+a_{z x}^{2}}
\end{gathered}
$$




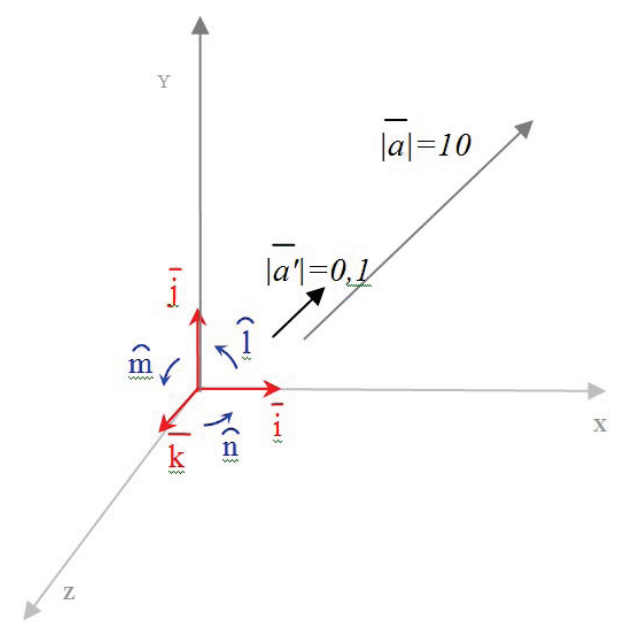

Fig. 7. Rectilinear inverse vector

\subsection{Multiplication of a Vector by a Scalar}

If all vectors in the equation are of the same type (rectilinear or angular), then to multiply a vector by a scalar all its projections are multiplied by the scalar

$$
n \bar{a}\left(n a_{x}, n a_{y}, n a_{z}\right)
$$

For all vectors multiplication by unit does not change the vector $\quad 1 \cdot \widehat{b}=\widehat{b}$

All vectors subject to the associativity property

$$
\lambda(\mu \widehat{b})=(\lambda \mu) \widehat{b}
$$

All vectors subject to the distributivity property

$$
(\lambda+\mu) \widehat{b}=\lambda \widehat{b}+\mu \widehat{b}
$$

Distributivity regarding the addition of vectors

$$
\lambda(\widehat{a}+\widehat{b})=\lambda \widehat{a}+\lambda \hat{b}
$$

\subsection{The Scalar Product of the Vectors}

From a physical point of view, the scalar product is the creation of a new physical quantity without direction. For example: $P=\bar{F} \cdot \bar{v}$ - power equals to the product of the speed and force $P=\widehat{M} \cdot \widehat{W}$ - power is equal to the product of the torque on the angular velocity

The basis of the scalar product is the product of two parallel vectors. And only a portion of the first vector participates in the product. It is that portion which can be represented by a parallel to the second vector (projection of the first vector on the second vector). Or vice versa.

Scalar product of two angular vectors is the number (scalar), equal to the product of the magnitude of angular vectors on the cosine of the angle between the planes, where the angular vectors are located.

$$
\widehat{a} \cdot \widehat{b}=|\widehat{a}| \cdot|\widehat{b}| \cdot \cos \angle(\widehat{a}, \widehat{b})=\widehat{a} \cdot b_{a}=a_{b} \cdot \widehat{b} \quad \text { - in vector form }
$$

Let us find the scalar product of the angular vector in the coordinate vector form:

Because the products of basis vectors in scalar product

$$
\begin{gathered}
\hat{l} \cdot \hat{l}=1, \widehat{l} \cdot \widehat{m}=0, \hat{m} \cdot \widehat{n}=0, \hat{n} \cdot \widehat{l}=0, \text { to } \\
\widehat{a} \cdot \widehat{b}=\left(a_{x y} \widehat{l}+a_{y z} \hat{m}+a_{z x} \widehat{n}\right) \cdot\left(b_{x y} \widehat{l}+b_{y z} \hat{m}+b_{z x} \widehat{n}\right)=
\end{gathered}
$$




$$
\begin{gathered}
=a_{x y} b_{x y}(\hat{l} \cdot \widehat{l})+a_{x y} b_{y z}(\hat{l} \cdot \widehat{m})+a_{x y} b_{z x}(\widehat{l} \cdot \widehat{n})+a_{y z} b_{x y}(\widehat{m} \cdot \widehat{l})+a_{y z} b_{y z}(\widehat{m} \cdot \widehat{m})+ \\
+a_{y z} b_{z x}(\widehat{m} \cdot \widehat{n})+a_{z x} b_{x y}(\hat{n} \cdot \widehat{l})+a_{z x} b_{y z}(\widehat{n} \cdot \widehat{m})+a_{z x} b_{z x}(\widehat{n} \cdot \widehat{n})=a_{x y} b_{x y}+a_{y z} b_{y z}+a_{z x} b_{z x} \\
\hat{a} \cdot \widehat{b}=a_{x y} b_{x y}+a_{y z} b_{y z}+a_{z x} b_{z x}
\end{gathered}
$$

It should be noted that, a scalar although it has no direction, it can be both positive and negative. And a scalar symbol does not denote the physical quantity direction but its condition (both for rectilinear and angular quantities).

For example: $P=\widehat{M} \cdot \widehat{w}=(-10 \cdot 100)+(0 \cdot 0)+(0 \cdot 0)=-1000(\mathrm{~W})$

This is the power of brake gear (or generator). It creates an inhibitory torque on the shaft $\widehat{M}=(-10,0,0)(\mathrm{Nm})$, which rotates with an angular speed $\widehat{w}=(100,0,0)(\mathrm{RAD} / \mathrm{s})$. The negative power can also e called a power of resistance.

\subsection{The Cross Product of the Vectors}

Mathematics is a universal discipline in which one and the same equation can be explained and applied differently. Let us demonstrate it on the following properties of the vectors.

The basis of the cross product of vectors is the product of two perpendicular vectors. And only a portion of the first vector participates in the product which can be represented by a perpendicular to the second vector (and vice versa), (Wilson, Edwin Bidwell, 1901).

$$
\bar{a} \times \bar{b}=|\bar{a}| \cdot|\bar{b}| \cdot \sin \angle(\bar{a}, \bar{b})=\bar{a} \cdot b_{p l \perp \bar{a}}=a_{p l \perp \bar{b}} \cdot \bar{b}
$$

$b_{p l \perp \bar{a}}$ - is the projection of the vector $\bar{b}$ onto the plane perpendicular to the vector $\bar{a}$

The result of the cross product of vectors is the formation of a new vector (new physical quantity). Consider 4 versions of cross products.

One must also remember that the chosen coordinate system affects the direction of rectilinear and angular vectors, respectively, it affects the output formula. Let us list these options involving unit vectors (basis vectors), for coordinate system in Figure 6, with a positive angular direction to coordinate planes $O x \rightarrow O y \rightarrow O z$ (counterclockwise):

1. The cross product of the two rectilinear vectors. The result is a rectilinear vector located perpendicularly to the outgoing vectors and directed by the right-hand rule (along the $\mathrm{z}$ axis, Figure 6).

$$
\bar{i} \times \bar{j}=\bar{k}, \quad \bar{j} \times \bar{k}=\bar{i}, \quad \bar{k} \times \bar{i}=\bar{j}
$$

2. The cross product of the two rectilinear vectors. The result is an angular vector located in the plane formed by the rectilinear outgoing vectors, and directed counterclockwise.

$$
\bar{i} \times \bar{j}=\widehat{l}, \quad \bar{j} \times \bar{k}=\widehat{m}, \quad \bar{k} \times \bar{i}=\widehat{n}
$$

3. The cross product of the two angular vectors. The result is an angular vector located in the plane perpendicular to the plane of outgoing angular vectors, and directed counterclockwise.

$$
\hat{l} \times \hat{m}=\widehat{n}, \quad \hat{m} \times \hat{n}=\hat{l}, \quad \hat{n} \times \hat{l}=\hat{m}
$$

4. The cross product of the two angular vectors. The result is a rectilinear vector, located at the intersection line of outgoing planes of angular vectors with positive touch direction of the plane of the second angular vector in the equation to the intersection line.

$$
\hat{l} \times \hat{m}=\bar{j}, \quad \hat{m} \times \hat{n}=\bar{k}, \quad \hat{n} \times \hat{l}=\bar{i}
$$

Let's consider each of the options of cross product and present them in a coordinate vector form:

1. $\bar{i} \times \bar{j}=\bar{k}, \quad \bar{j} \times \bar{k}=\bar{i}, \quad \bar{k} \times \bar{i}=\bar{j}$ cross product of the two rectilinear vectors. The result is a rectilinear vectors.

The cross product of the vectors is well known to all, but to compare it with other cross products, we also consider it.

Let us find the distribution of the resulting rectilinear vector on coordinate axes:

$$
\bar{b}=\bar{c} \times \bar{d}=\left(c_{x} \bar{i}+c_{y} \bar{j}+c_{z} \bar{k}\right) \times\left(d_{x} \bar{i}+d_{y} \bar{j}+d_{z} \bar{k}\right)=
$$




$$
\begin{gathered}
=c_{x} d_{x}(\bar{i} \times \bar{i})+c_{x} d_{y}(\bar{i} \times \bar{j})+c_{x} d_{z}(\bar{i} \times \bar{k})+c_{y} d_{x}(\bar{j} \times \bar{i})+c_{y} d_{y}(\bar{j} \times \bar{j})+c_{y} d_{z}(\bar{j} \times \bar{k})+ \\
+c_{z} d_{x}(\bar{k} \times \bar{i})+c_{z} d_{y}(\bar{k} \times \bar{j})+c_{z} d_{z}(\bar{k} \times \bar{k})=0+c_{x} d_{y}(\bar{k})-c_{x} d_{z}(\bar{j})-c_{y} d_{x}(\bar{k})+0+c_{y} d_{z}(\bar{i})+ \\
+c_{z} d_{x}(\bar{j})-c_{z} d_{y}(\bar{i})+0=\left(c_{y} d_{z}-c_{z} d_{y}\right) \bar{i}+\left(c_{z} d_{x}-c_{x} d_{z}\right) \bar{j}+\left(c_{x} d_{y}-c_{y} d_{x}\right) \bar{k}=b_{x} \bar{i}+b_{y} \bar{j}+b_{z} \bar{k} \\
b_{x}=c_{y} d_{z}-c_{z} d_{y}, \quad b_{y}=c_{z} d_{x}-c_{x} d_{z}, \quad b_{z}=c_{x} d_{y}-c_{y} d_{x}
\end{gathered}
$$

2. $\bar{i} \times \bar{j}=\hat{l}, \quad \bar{j} \times \bar{k}=\widehat{m}, \quad \bar{k} \times \bar{i}=\widehat{n}$ is the cross product of the two rectilinear vectors is an angular vector.

Let us find the distribution of the resulting angular vector on coordinate plane (present it in coordinate-vector form),

$$
\begin{gathered}
\hat{b}=\bar{c} \times \bar{d}=\left(c_{x} \bar{i}+c_{y} \bar{j}+c_{z} \bar{k}\right) \times\left(d_{x} \bar{i}+d_{y} \bar{j}+d_{z} \bar{k}\right)= \\
=c_{x} d_{x}(\bar{i} \times \bar{i})+c_{x} d_{y}(\bar{i} \times \bar{j})+c_{x} d_{z}(\bar{i} \times \bar{k})+c_{y} d_{x}(\bar{j} \times \bar{i})+c_{y} d_{y}(\bar{j} \times \bar{j})+c_{y} d_{z}(\bar{j} \times \bar{k})+ \\
+c_{z} d_{x}(\bar{k} \times \bar{i})+c_{z} d_{y}(\bar{k} \times \bar{j})+c_{z} d_{z}(\bar{k} \times \bar{k})=0+c_{x} d_{y}(\widehat{l})-c_{x} d_{z}(\widehat{n})-c_{y} d_{x}(\widehat{l})+0+c_{y} d_{z}(\widehat{m})+ \\
+c_{z} d_{x}(\widehat{n})-c_{z} d_{y}(\widehat{m})+0=\left(c_{x} d_{y}-c_{y} d_{x}\right) \hat{l}+\left(c_{y} d_{z}-c_{z} d_{y}\right) \hat{m}+\left(c_{z} d_{x}-c_{x} d_{z}\right) \hat{n}=b_{x y} \hat{l}+b_{y z} \hat{m}+b_{z x} \widehat{n} \\
b_{x y}=c_{x} d_{y}-c_{y} d_{x}, \quad b_{y z}=c_{y} d_{z}-c_{z} d_{y}, \quad b_{z x}=c_{z} d_{x}-c_{x} d_{z}
\end{gathered}
$$

3. $\hat{l} \times \hat{m}=\widehat{n}, \quad \hat{m} \times \hat{n}=\hat{l}, \quad \hat{n} \times \hat{l}=\hat{m}$ the cross product of two angular vectors is the 3rd angular vector perpendicular to outgoing vectors.

Let us find the distribution of the resulting angular vector on coordinate planes,

$$
\begin{aligned}
& \widehat{b}=\widehat{c} \times \widehat{d}=\left(c_{x y} \hat{l}+c_{y z} \hat{m}+c_{z x} \widehat{n}\right) \times\left(d_{x y} \hat{l}+d_{y z} \hat{m}+d_{z x} \widehat{n}\right)= \\
& =c_{x y} d_{x y}(\hat{l} \times \hat{l})+c_{x y} d_{y z}(\hat{l} \times \widehat{m})+c_{x y} d_{z x}(\hat{l} \times \widehat{n})+c_{y z} d_{x y}(\hat{m} \times \hat{l})+c_{y z} d_{y z}(\hat{m} \times \widehat{m})+ \\
& +c_{y z} d_{z x}(\hat{m} \times \widehat{n})+c_{z x} d_{x y}(\hat{n} \times \widehat{l})+c_{z x} d_{y z}(\widehat{n} \times \widehat{m})+c_{z x} d_{z x}(\widehat{n} \times \widehat{n})=0+c_{x y} d_{y z}(\widehat{n})- \\
& -c_{x y} d_{z x}(\widehat{m})-c_{y z} d_{x y}(\widehat{n})+0+c_{y z} d_{z x}(\widehat{l})+c_{z x} d_{x y}(\widehat{m})-c_{z x} d_{y z}(\widehat{l})+0= \\
& =\left(c_{y z} d_{z x}-c_{z x} d_{y z}\right) \hat{l}+\left(c_{z x} d_{x y}-c_{x y} d_{z x}\right) \hat{m}+\left(c_{x y} d_{y z}-c_{y z} d_{x y}\right) \hat{n}=b_{x y} \hat{l}+b_{y z} \hat{m}+b_{z x} \widehat{n} \\
& b_{x y}=c_{y z} d_{z x}-c_{z x} d_{y z}, \quad b_{y z}=c_{z x} d_{x y}-c_{x y} d_{z x}, \quad b_{z x}=c_{x y} d_{y z}-c_{y z} d_{x y}
\end{aligned}
$$

4. $\hat{l} \times \hat{m}=\bar{j}, \quad \hat{m} \times \hat{n}=\bar{k}, \quad \hat{n} \times \hat{l}=\bar{i}$ the cross product of two angular vectors is rectilinear vector.

Let us find the distribution of the resulting angular vector on coordinate axis,

$$
\begin{aligned}
& \bar{b}=\widehat{c} \times \widehat{d}=\left(c_{x y} \hat{l}+c_{y z} \hat{m}+c_{z x} \hat{n}\right) \times\left(d_{x y} \hat{l}+d_{y z} \hat{m}+d_{z x} \widehat{n}\right)= \\
& =c_{x y} d_{x y}(\hat{l} \times \hat{l})+c_{x y} d_{y z}(\hat{l} \times \widehat{m})+c_{x y} d_{z x}(\hat{l} \times \widehat{n})+c_{y z} d_{x y}(\hat{m} \times \hat{l})+c_{y z} d_{y z}(\hat{m} \times \widehat{m})+ \\
& +c_{y z} d_{z x}(\hat{m} \times \widehat{n})+c_{z x} d_{x y}(\hat{n} \times \hat{l})+c_{z x} d_{y z}(\hat{n} \times \widehat{m})+c_{z x} d_{z x}(\hat{n} \times \widehat{n})=0+c_{x y} d_{y z}(\bar{j})- \\
& -c_{x y} d_{z x}(\bar{i})-c_{y z} d_{x y}(\bar{j})+0+c_{y z} d_{z x}(\bar{k})+c_{z x} d_{x y}(\bar{i})-c_{z x} d_{y z}(\bar{k})+0= \\
& =\left(c_{z x} d_{x y}-c_{x y} d_{z x}\right) \bar{i}+\left(c_{x y} d_{y z}-c_{y z} d_{x y}\right) \bar{j}+\left(c_{y z} d_{z x}-c_{z x} d_{y z}\right) \bar{k}=b_{x} \bar{i}+b_{y} \bar{j}+b_{z} \bar{k} \\
& b_{x}=c_{z x} d_{x y}-c_{x y} d_{z x}, \quad b_{y}=c_{x y} d_{y z}-c_{y z} d_{x y}, \quad b_{z}=c_{y z} d_{z x}-c_{z x} d_{y z}
\end{aligned}
$$

Remember that the cross product of the vectors of the same type is anticommutative

$$
\begin{gathered}
\bar{c}=\bar{a} \times \bar{b}=-(\bar{b} \times \bar{a}) \\
\widehat{c}=\bar{a} \times \bar{b}=-(\bar{b} \times \bar{a}) \\
\bar{c}=\widehat{a} \times \widehat{b}=-(\widehat{b} \times \widehat{a}) \\
\widehat{c}=\widehat{a} \times \widehat{b}=-(\widehat{b} \times \widehat{a})
\end{gathered}
$$




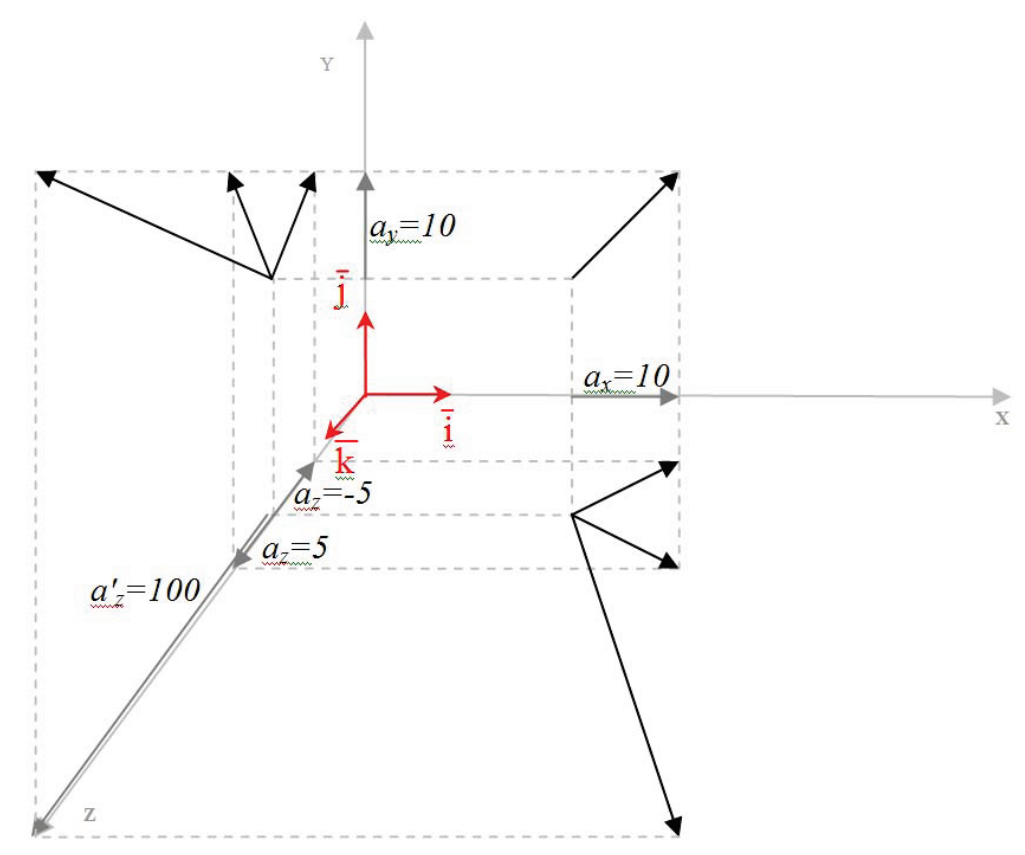

Fig. 8. Projections of the rectilinear vectors in planes and axes

Which of these equations have a right to exist?

For this let's consider two samples, which will show that cross product of the vectors $\bar{i} \times \bar{j}=\bar{k}, \hat{l} \times \hat{m}=\hat{n}$ cannot exist.

Example 3: Let us assume that the modulus of rectilinear vector $|\bar{a}|=15$ and two of its projections onto the coordinate axes $\bar{a}\left(10,10, a_{z}\right)$, fig. 8 (the vector itself $\bar{a}$ is not shown). One must find its third projection.

Solution:

Using the formula $|\bar{a}|=\sqrt{a_{x}^{2}+a_{y}^{2}+a_{z}^{2}}$, the projection of the vector is equal

$$
a_{z}=\sqrt{15^{2}-10^{2}-10^{2}}= \pm 5
$$

It is impossible to say more exactly in what side the vector projection is directed on an axis Oz. Now present our vector as sum of coordinate vectors.

$$
\bar{a}\left(a_{x}, a_{y}, a_{z}\right)=a_{x} \bar{i}+a_{y} \bar{j}+a_{z} \bar{k}=\bar{a}_{x}+\bar{a}_{y}+\bar{a}_{z}, \quad \text { then } \quad \bar{a}_{x}=a_{x}, \quad \bar{a}_{y}=a_{y}, \quad \bar{a}_{z}=a_{z}
$$

and using the formula $\bar{i} \times \bar{j}=\bar{k}$ (32), let us find the projection of the vector $a_{z}$, naming it $a_{z}^{\prime}$

$$
\begin{gathered}
\bar{a}_{z}^{\prime}=\bar{a}_{x} \times \bar{a}_{y}=\left(a_{x} \bar{i}+0+0\right) \times\left(0+a_{y} \bar{j}+0\right)=0 \bar{i}+0 \bar{j}+\left(a_{x} a_{y}-0\right) \bar{k}=0 \bar{i}+0 \bar{j}+a_{z} \bar{k} \\
a_{z}^{\prime}=a_{x} a_{y}=10 \cdot 10=100
\end{gathered}
$$

Conclusions: Rectilinear vector $a_{z}^{\prime}$ projection found by the second method:

1. is not consistent with the projection of the vector $a_{z}$ found by the first method, $a_{z} \neq a_{z}^{\prime}$

2. is not consistent with the modulus of the specified vector, if one will find modulus using $a_{z}^{\prime}$

3. specifies only a positive direction, although the direction is not precisely defined and can be both positive and negative in the $\mathrm{Oz}$ axis.

Thus, the cross product $\bar{i} \times \bar{j}=\bar{k}$ is not consistent with the other properties of the rectilinear vectors, and has no right to exist.

Similar to this example, one can prove that the cross product $\hat{l} \times \hat{m}=\hat{n}$ does not exist.

Example 4: One should pay attention to another problem - the logical inconsistency of the cross product of vectors $\bar{i} \times \bar{j}=\bar{k}$. 
With the cross product of two rectilinear vectors, a new rectilinear vector is created which is perpendicular to both original vectors $\bar{d}=\bar{b} \times \bar{c}$. It would be logical if $\bar{b}=\bar{c} \times \bar{d}$ and $\bar{c}=\bar{d} \times \bar{b}$. That is, knowing any two vectors, one could find a third vector. But this is not observed (observed only if one presents the vectors as unit vectors).

For example, if there are two rectilinear vectors $\bar{b}(10,0,0), \bar{c}(0,10,0)$, then

$$
\begin{gathered}
\bar{d}=\bar{b} \times \bar{c}=\left(b_{y} c_{z}-b_{z} c_{y}\right) \bar{i}+\left(b_{z} c_{x}-b_{x} c_{z}\right) \bar{j}+\left(b_{x} c_{y}-b_{y} c_{x}\right) \bar{k}=d_{x} \bar{i}+d_{y} \bar{j}+d_{z} \bar{k} \\
\bar{d}=(0 \cdot 0-0 \cdot 10) \bar{i}+(0 \cdot 0-10 \cdot 0) \bar{j}+(10 \cdot 10-0 \cdot 0) \bar{k}=0 \bar{i}+0 \bar{j}+100 \bar{k}
\end{gathered}
$$

$d_{x}=0, d_{y}=0, d_{z}=100$ that is, the vector $\bar{d}(0,0,100)$

Now we find the vector $\bar{b}$ again and compare it with the original vector

$$
\begin{aligned}
& \bar{b}=\bar{c} \times \bar{d}=\left(c_{y} d_{z}-c_{z} d_{y}\right) \bar{i}+\left(c_{z} d_{x}-c_{x} d_{z}\right) \bar{j}+\left(c_{x} d_{y}-c_{y} d_{x}\right) \bar{k}=b_{x} \bar{i}+b_{y} \bar{j}+b_{z} \bar{k} \\
& \bar{b}=(10 \cdot 100-0 \cdot 0) \bar{i}+(0 \cdot 0-0 \cdot 100) \bar{j}+(0 \cdot 0-10 \cdot 0) \bar{k}=1000 \bar{i}+0 \bar{j}+0 \bar{k}
\end{aligned}
$$

$b_{x}=1000, b_{y}=0, b_{z}=0$, that is there is a vector $\bar{b}(1000,0,0)$, and is not equal to the original vector.

This observation is true for the cross product of angular vectors $\widehat{d}=\widehat{b} \times \widehat{c}$, as a result of which the third angular vector is formed.

Consider the following example, which shows that the cross product $\bar{i} \times \bar{j}=\hat{l}$ has the right to exist.

\section{Example 5:}

Let us assume that two rectilinear vectors are given $\bar{a}(3,-4,5), \bar{b}(-5,2,-8)$, fig.9.

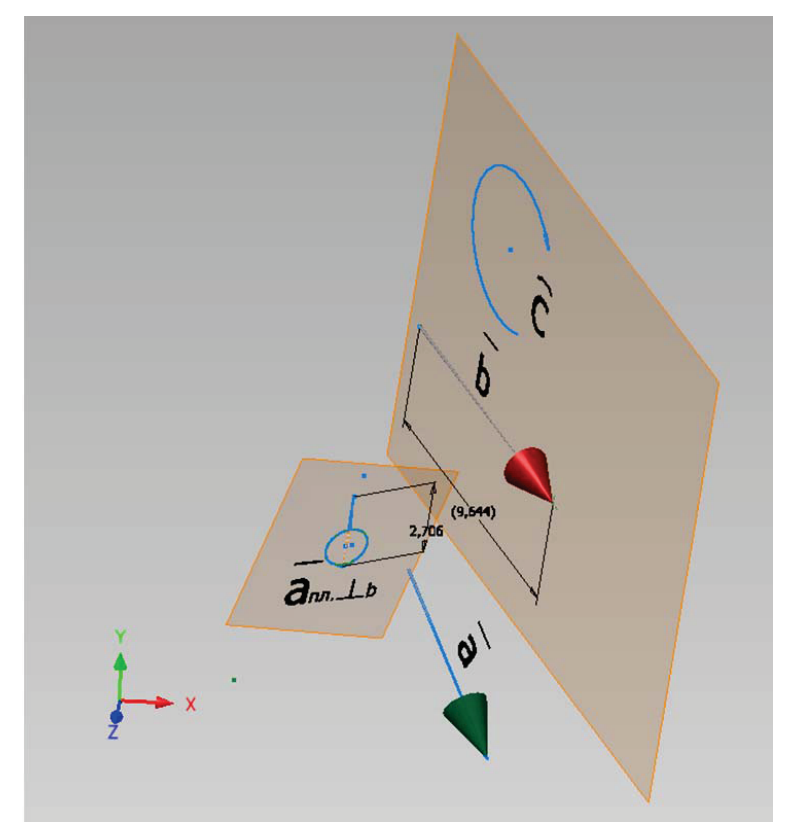

Fig. 9. Rectilinear and angular vectors in coordinate system

Let us find the angular vector $\widehat{c}$ formed by these rectilinear vectors (33):

$$
\begin{gathered}
\widehat{c}=\bar{a} \times \bar{b}=\left(a_{x} b_{y}-a_{y} b_{x}\right) \hat{l}+\left(a_{y} b_{z}-a_{z} b_{y}\right) \hat{m}+\left(a_{z} b_{x}-a_{x} b_{z}\right) \hat{n}=c_{x y} \hat{l}+c_{y z} \hat{m}+c_{z x} \hat{n} \\
c_{x y}=a_{x} b_{y}-a_{y} b_{x}=3 \cdot 2-(-4) \cdot(-5)=-14 \\
c_{y z}=a_{y} b_{z}-a_{z} b_{y}=(-4) \cdot(-8)-5 \cdot 2=22 \\
c_{z x}=a_{z} b_{x}-a_{x} b_{z}=5 \cdot(-5)-3 \cdot(-8)=-1 \\
\hat{c}(-14,22,-1)
\end{gathered}
$$


Then angular vector modulus

$$
|\widehat{c}|=\sqrt{c_{x y}^{2}+c_{y z}^{2}+c_{z x}^{2}}=\sqrt{(-14)^{2}+(22)^{2}+(-1)^{2}}=\sqrt{196+484+1}=26,09
$$

Let us find the modulus in a different way, to do this, we should find first the moduluses of rectilinear vectors:

$$
\begin{aligned}
& |\bar{a}|=\sqrt{a_{x}^{2}+a_{y}^{2}+a_{z}^{2}}=\sqrt{(3)^{2}+(-4)^{2}+(5)^{2}}=\sqrt{9+16+25}=7,071 \\
& |\bar{b}|=\sqrt{b_{x}^{2}+b_{y}^{2}+b_{z}^{2}}=\sqrt{(-5)^{2}+(2)^{2}+(-8)^{2}}=\sqrt{25+4+64}=9,64
\end{aligned}
$$

As this is an angular vector this cross product of two rectilinear vectors, present vector $\bar{a}$ as a projection of the vector onto the plane perpendicular to the vector $\bar{b}$, fig.9. And by using Autodesk Inventor program, let us find the magnitude of the projection of the vector $\bar{a}_{p l \perp \bar{b}}=2,706$.

Then angular vector modulus $|\widehat{c}|=|\bar{b}| \cdot\left|\bar{a}_{p l \perp \bar{b}}\right|=9,64 \cdot 2,706=26,085$

In this equation, everything is taken in modulus, as since the angular vector $\widehat{c}$ is in space, and we can not know its direction. The direction of the angular vector is determined through its projections onto the coordinate planes $\widehat{c}\left(c_{x y}, c_{y z}, c_{z x}\right)$, as the direction of the vectors is specified only by the coordinate system.

If you combine the end of the projection of the vector $\bar{a}_{p l \perp \bar{b}}$ with the beginning of the vector $\bar{b}$, they will form a plane (let's name it as the first plane $p l .(1) \widehat{c})$ in which the angular vector $\widehat{c}$ is located.

On the contrary, if we find the projection of the vector $\bar{b}_{p l \perp \bar{a}}=3,691$ onto the plane perpendicular to the vector $\bar{a}$, then the angular vector

$$
|\widehat{c}|=|\bar{a}| \cdot\left|\bar{b}_{p l \perp \bar{a}}\right|=7,071 \cdot 3,691=26,099 .
$$

Plane of angular vector $\widehat{c}$ can also be presented out of straight line of the vector $\bar{a}$, and vector projection $\bar{b}_{p l \perp \bar{a}}$ on perpendicular to the plane. Let's name it as the second plane of angular vector $p l .(2) \hat{c}$.

Analyzing these planes, we can say that they are parallel. This is logical because the angular vector can be one of the many parallel, like-directed angular vectors.

Conclusions: modulus of the angular vector $|\widehat{c}|$, found by two ways, is the same (taking into account the error margin of used Autodesk Inventor programme).

Two examples where the cross product $\bar{i} \times \bar{j}=\hat{l}$ occurs in nature.

\section{Example 6:}

Let us find a decomposition of the torque on the coordinate planes. At the positive angular direction of the torque in the axes $O x \longrightarrow O y \longrightarrow O z:$

$$
\begin{gathered}
\bar{i} \times \bar{j}=\widehat{l}, \quad \bar{j} \times \bar{k}=\widehat{m}, \quad \bar{k} \times \bar{i}=\widehat{n} \\
\widehat{M}=\bar{h} \times \bar{F}=\left(h_{x} \bar{i}+h_{y} \bar{j}+h_{z} \bar{k}\right) \times\left(F_{x} \bar{i}+F_{y} \bar{j}+F_{z} \bar{k}\right)= \\
=h_{x} F_{x}(\bar{i} \times \bar{i})+h_{x} F_{y}(\bar{i} \times \bar{j})+h_{x} F_{z}(\bar{i} \times \bar{k})+h_{y} F_{x}(\bar{j} \times \bar{i})+h_{y} F_{y}(\bar{j} \times \bar{j})+h_{y} F_{z}(\bar{j} \times \bar{k})+ \\
+h_{z} F_{x}(\bar{k} \times \bar{i})+h_{z} F_{y}(\bar{k} \times \bar{j})+h_{z} F_{z}(\bar{k} \times \bar{k})=0+h_{x} F_{y}(\widehat{l})-h_{x} F_{z}(\widehat{n})-h_{y} F_{x}(\widehat{l})+0+h_{y} F_{z}(\widehat{m})+ \\
+h_{z} F_{x}(\widehat{n})-h_{z} F_{y}(\hat{m})+0=\left(h_{x} F_{y}-h_{y} F_{x}\right) \hat{l}+\left(h_{y} F_{z}-h_{z} F_{y}\right) \hat{m}+\left(h_{z} F_{x}-h_{x} F_{z}\right) \widehat{n}= \\
=M_{x y} \hat{l}+M_{y z} \hat{m}+M_{z x} \hat{n},
\end{gathered}
$$

then

$$
M_{x y}=h_{x} F_{y}-h_{y} F_{x}, \quad M_{y z}=h_{y} F_{z}-h_{z} F_{y}, \quad M_{z x}=h_{z} F_{x}-h_{x} F_{z}
$$

Conclusions: In this cross product of rectilinear vectors distances and forces are in the same plane with angular vector of torque. Their direction and magnitude are aligned with physical quantities.

Example 7: Let us find a decomposition of the angular velocity on the coordinate planes. 
At the positive angular direction of the angular velocity in the axes $O x \longrightarrow O y \longrightarrow O z$ :

$$
\bar{i} \times \bar{j}=\hat{l}, \quad \bar{j} \times \bar{k}=\widehat{m}, \quad \bar{k} \times \bar{i}=\hat{n}
$$

Let us transform the equation of the tangential velocity $\bar{v}=\bar{r} \times \widehat{w}$, in the equation of the angular velocity $\frac{1}{\bar{r}} \times \bar{v}=\widehat{w}$

Rectilinear vector of distance is represented as inverse vector in cross product of vectors, then

$$
\begin{gathered}
\widehat{w}=\frac{1}{\bar{r}} \times \bar{v}=\overline{r^{\prime}} \times \bar{v}=\left(r_{x}^{\prime} \bar{i}+r_{y}^{\prime} \bar{j}+r_{z}^{\prime} \bar{k}\right) \times\left(v_{x} \bar{i}+v_{y} \bar{j}+v_{z} \bar{k}\right)= \\
=r_{x}^{\prime} v_{x}(\bar{i} \times \bar{i})+r_{x}^{\prime} v_{y}(\bar{i} \times \bar{j})+r_{x}^{\prime} v_{z}(\bar{i} \times \bar{k})+r_{y}^{\prime} v_{x}(\bar{j} \times \bar{i})+r_{y}^{\prime} v_{y}(\bar{j} \times \bar{j})+r_{y}^{\prime} v_{z}(\bar{j} \times \bar{k})+r_{z}^{\prime} v_{x}(\bar{k} \times \bar{i})+r_{z}^{\prime} v_{y}(\bar{k} \times \bar{j})+r_{z}^{\prime} v_{z}(\bar{k} \times \bar{k})= \\
=0+r_{x}^{\prime} v_{y}(\hat{l})-r_{x}^{\prime} v_{z}(\widehat{n})-r_{y}^{\prime} v_{x}(\hat{l})+0+r_{y}^{\prime} v_{z}(\widehat{m})+r_{z}^{\prime} v_{x}(\widehat{n})-r_{z}^{\prime} v_{y}(\widehat{m})+0=\left(r_{x}^{\prime} v_{y}-r_{y}^{\prime} v_{x}\right) \hat{l}+\left(r_{y}^{\prime} v_{z}-r_{z}^{\prime} v_{y}\right) \hat{m}+\left(r_{z}^{\prime} v_{x}-r_{x}^{\prime} v_{z}\right) \hat{n}= \\
=\left(\frac{r_{x} v_{y}}{r_{x}^{2}+r_{y}^{2}+r_{z}^{2}}-\frac{r_{y} v_{x}}{r_{x}^{2}+r_{y}^{2}+r_{z}^{2}}\right) \hat{l}+\left(\frac{r_{y} v_{z}}{r_{x}^{2}+r_{y}^{2}+r_{z}^{2}}-\frac{r_{z} v_{y}}{r_{x}^{2}+r_{y}^{2}+r_{z}^{2}}\right) \hat{m}+\left(\frac{r_{z} v_{x}}{r_{x}^{2}+r_{y}^{2}+r_{z}^{2}}-\frac{r_{x}}{r_{x}^{2}+r_{y}^{2}+r_{z}^{2}}\right) \widehat{n}= \\
=\left(\frac{r_{x} v_{y}-r_{y} v_{x}}{r_{x}^{2}+r_{y}^{2}+r_{z}^{2}}\right) \widehat{l}+\left(\frac{r_{y} v_{z}-r_{z} v_{y}}{r_{x}^{2}+r_{y}^{2}+r_{z}^{2}}\right) \hat{m}+\left(\frac{r_{z} v_{x}-r_{x} v_{z}}{r_{x}^{2}+r_{y}^{2}+r_{z}^{2}}\right) \widehat{n}=w_{x y} \hat{l}+w_{y z} \hat{m}+w_{z x} \hat{n},
\end{gathered}
$$

then

$$
w_{x y}=\frac{r_{x} v_{y}-r_{y} v_{x}}{r_{x}^{2}+r_{y}^{2}+r_{z}^{2}}, \quad w_{y z}=\frac{r_{y} v_{z}-r_{z} v_{y}}{r_{x}^{2}+r_{y}^{2}+r_{z}^{2}}, \quad w_{z x}=\frac{r_{z} v_{x}-r_{x} v_{z}}{r_{x}^{2}+r_{y}^{2}+r_{z}^{2}}
$$

Conclusions: in this cross product of rectilinear vectors distances and tangential velocities are in the same plane with angular velocity vector. Their direction and magnitude are aligned with physical quantities.

Now let us prove that the cross product $\hat{l} \times \widehat{m}=\bar{j}$, which forms a rectilinear vector of two angular vectors, does not exist.

Example 8: Let us assume that two rectilinear vectors $\bar{a}(10,0,0), \bar{b}(0,10,0), \bar{c}(0,0,10)$, are given, fig. 10 . Let us find the angular vectors formed of these linear vectors in the plane $O X Y, O Y Z$.

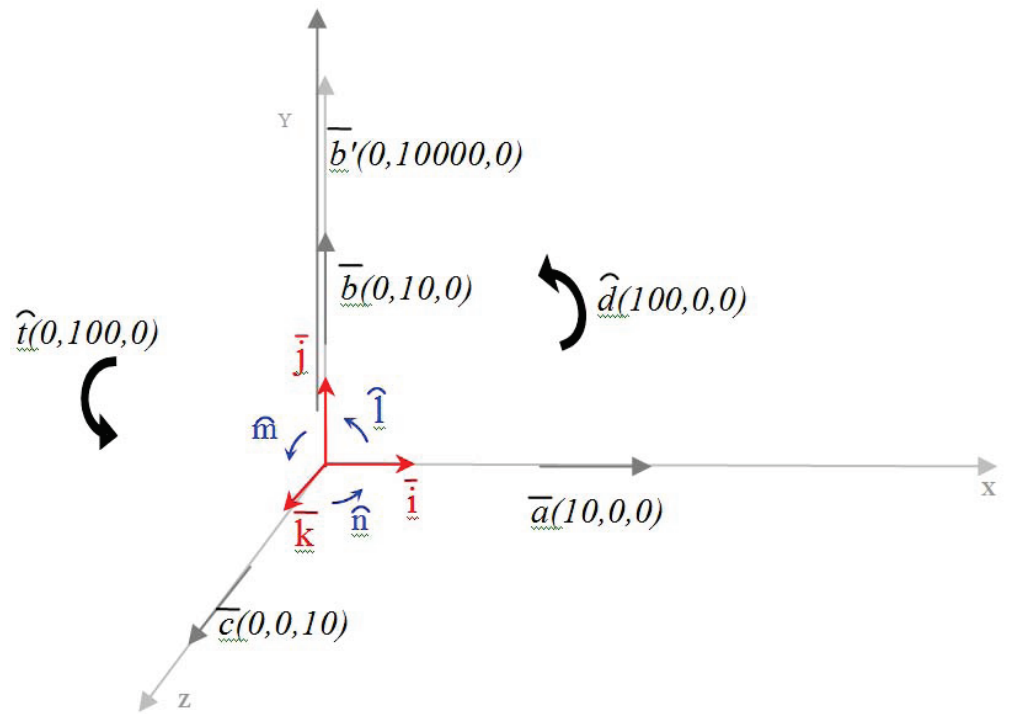

Fig. 10. Rectilinear and angular vectors in coordinate system

$$
\begin{gathered}
\hat{d}=\bar{a} \times \bar{b}=\left(a_{x} b_{y}-a_{y} b_{x}\right) \hat{l}+\left(a_{y} b_{z}-a_{z} b_{y}\right) \hat{m}+\left(a_{z} b_{x}-a_{x} b_{z}\right) \hat{n}=d_{x y} \widehat{l}+d_{y z} \hat{m}+d_{z x} \hat{n} \\
\hat{d}=(10 \cdot 10-0 \cdot 0) \hat{l}+(0 \cdot 0-0 \cdot 10) \hat{m}+(0 \cdot 0-10 \cdot 0) \hat{n}=100 \hat{l}+0 \hat{m}+0 \hat{n} \\
\hat{d}(100,0,0) \\
\hat{t}=\bar{b} \times \bar{c}=\left(b_{x} c_{y}-b_{y} c_{x}\right) \hat{l}+\left(b_{y} c_{z}-b_{z} c_{y}\right) \hat{m}+\left(b_{z} c_{x}-b_{x} c_{z}\right) \hat{n}=t_{x y} \hat{l}+t_{y z} \hat{m}+t_{z x} \hat{n}
\end{gathered}
$$




$$
\begin{gathered}
\widehat{t}=(0 \cdot 0-10 \cdot 0) \hat{l}+(10 \cdot 10-0 \cdot 0) \hat{m}+(0 \cdot 0-0 \cdot 10) \hat{n}=0 \hat{l}+100 \hat{m}+0 \hat{n} \\
\widehat{t}(0,100,0)
\end{gathered}
$$

Let us find a rectilinear vector $\overline{b^{\prime}}$ of angular vectors $\widehat{d}, \widehat{t}$ and compare it with the specified vector $\bar{b}$.

$$
\begin{gathered}
\overline{b^{\prime}}=\hat{d} \times \hat{t}=\left(d_{z x} t_{x y}-d_{x y} t_{z x}\right) \bar{i}+\left(d_{x y} t_{y z}-d_{y z} t_{x y}\right) \bar{j}+\left(d_{y z} t_{z x}-d_{z x} t_{y z}\right) \bar{k}=b_{x}^{\prime} \bar{i}+b_{y}^{\prime} \bar{j}+b_{z}^{\prime} \bar{k} \\
\overline{b^{\prime}}=(0 \cdot 0-100 \cdot 0) \bar{i}+(100 \cdot 100-0 \cdot 0) \bar{j}+(0 \cdot 0-0 \cdot 100) \bar{k}=0 \bar{i}+10000 \bar{j}+0 \bar{k} \\
\overline{b^{\prime}}(0,10000,0)
\end{gathered}
$$

Since vectors $\bar{b} \neq \overline{b^{\prime}}$ are not equal, then the cross product $\hat{l} \times \widehat{m}=\bar{j}$ may not exist.

The general conclusion of the cross products of the vectors: in this type of the product only one option is possible when at the cross product of two rectilinear vectors angular vector is formed $\bar{i} \times \bar{j}=\widehat{l}$.

The proof of this statement:

1. Rectilinear and angular vectors lie in one plane, that is the first necessary but not sufficient connection of the rectilinear and angular vectors.

2. The magnitude of the cross product of rectilinear vectors is equal to the area of the figure, and the magnitude of the angular vector is definitionally equal to the area of the figure.

3. The magnitude of the cross product of rectilinear vectors coincides with the magnitude of the angular vector. This is a consequence of observation during the formation of angular physical quantities (running torque, angular velocity).

4. Only two directions $(+,-)$ can be deduced from the cross product of rectilinear vectors. The angular vector also has two directions only.

5. The sequence of rectilinear vectors in the cross product definitely determines the direction of the angular vector in a plane.

\subsection{Scalar Product of the Mixed Vectors}

Scalar product of angular and rectilinear vectors is the number (scalar), equal to the product of the quantities of these vectors to the cosine of the smallest angle formed by the plane in which the angular vector is located, and the right line of the rectilinear vector. If both vectors are in the same plane, the scalar is equal to the product of their quantities.

$$
\bar{a} \cdot \widehat{b}=|\bar{a}| \cdot|\widehat{b}| \cdot|\cos (\bar{a}, \widehat{b})|=\left|a_{b}\right| \cdot|\widehat{b}| \quad \text { - in vector form }
$$

In this equation the scalar looses its sign and can be only positive.

Because the projection of the rectilinear vector on a plane does not determine its direction relative to the angular vector which is in this plane.

Deduce the equation in coordinate-vector form.

Because all products of the basis vectors are at scalar product

$$
\begin{aligned}
& \bar{i} \cdot \bar{i}=1, \quad \bar{j} \cdot \bar{j}=1, \quad \bar{k} \cdot \bar{k}=1, \\
& \bar{i} \cdot \bar{j}=0, \quad \bar{j} \cdot \bar{k}=0, \quad \bar{k} \cdot \bar{i}=0, \\
& \bar{i} \cdot \hat{l}=1, \quad \bar{j} \cdot \hat{l}=1, \quad \bar{k} \cdot \hat{l}=0, \\
& \bar{j} \cdot \widehat{m}=1, \quad \bar{k} \cdot \widehat{m}=1, \quad \bar{i} \cdot \widehat{m}=0, \\
& \bar{k} \cdot \hat{n}=1, \quad \bar{i} \cdot \widehat{n}=1, \quad \bar{j} \cdot \widehat{n}=0, \\
& c=\bar{a} \cdot \widehat{b}=\left(a_{x} \bar{i}+a_{y} \bar{j}+a_{z} \bar{k}\right) \cdot\left(b_{x y} \widehat{l}+b_{y z} \widehat{m}+b_{z x} \widehat{n}\right)= \\
& =a_{x} b_{x y}(\bar{i} \cdot \widehat{l})+a_{x} b_{y z}(\bar{i} \cdot \widehat{m})+a_{x} b_{z x}(\bar{i} \cdot \widehat{n})+a_{y} b_{x y}(\bar{j} \cdot \widehat{l})+a_{y} b_{y z}(\bar{j} \cdot \widehat{m})+a_{y} b_{z x}(\bar{j} \cdot \widehat{n})+a_{z} b_{x y}(\bar{k} \cdot \widehat{l})+a_{z} b_{y z}(\bar{k} \cdot \widehat{m})+a_{z} b_{z x}(\bar{k} \cdot \widehat{n})= \\
& =b_{x y} \hat{l}\left(a_{x} \bar{i}+a_{y} \bar{j}\right)+0+b_{y z} \widehat{m}\left(a_{y} \bar{j}+a_{z} \bar{k}\right)+0+b_{z x} \widehat{n}\left(a_{z} \bar{k}+a_{x} \bar{i}\right)+0= \\
& =b_{x y} \hat{l} \sqrt{\left(a_{x} \bar{i}+a_{y} \bar{j}\right)^{2}}+b_{y z} \hat{m} \sqrt{\left(a_{y} \bar{j}+a_{z} \bar{k}\right)^{2}}+b_{z x} \hat{n} \sqrt{\left(a_{z} \bar{k}+a_{x} \bar{i}\right)^{2}}=
\end{aligned}
$$




$$
\begin{gathered}
=b_{x y} \hat{l} \sqrt{a_{x}^{2} \bar{i}^{2}+2 a_{x} a_{y} \bar{i} \bar{j}+a_{y}^{2} \bar{j}^{2}}+b_{y z} \hat{m} \sqrt{a_{y}^{2} \bar{j}^{2}+2 a_{y} a_{z} \bar{j} \bar{k}+a_{z}^{2} \bar{k}^{2}}+b_{z x} \hat{n} \sqrt{a_{z}^{2} \bar{k}^{2}+2 a_{z} a_{x} \bar{k} \bar{i}+a_{x}^{2} \bar{i}^{2}}= \\
=b_{x y} \hat{l} \sqrt{a_{x}^{2} \bar{i}^{2}+a_{y}^{2} \bar{j}^{2}}+b_{y z} \hat{m} \sqrt{a_{y}^{2} \bar{j}^{2}+a_{z}^{2} \bar{k}^{2}}+b_{z x} \widehat{n} \sqrt{a_{z}^{2} \bar{k}^{2}+a_{x}^{2} \bar{i}^{2}}= \\
=b_{x y} \sqrt{a_{x}^{2}+a_{y}^{2}}+b_{y z} \sqrt{a_{y}^{2}+a_{z}^{2}}+b_{z x} \sqrt{a_{z}^{2}+a_{x}^{2}} \\
c=\left|b_{x y} \sqrt{a_{x}^{2}+a_{y}^{2}}+b_{y z} \sqrt{a_{y}^{2}+a_{z}^{2}}+b_{z x} \sqrt{a_{z}^{2}+a_{x}^{2}}\right|
\end{gathered}
$$

The equation is taken modulo because directions of angular and rectilinear vectors are not consistent.

2.9 Cross product of the Mixed vectors

Cross product of Mixed vectors is found from the equation (29)

$$
\begin{array}{ccc}
\bar{i} \times \bar{j}=\hat{l}, & \bar{j} \times \bar{k}=\widehat{m}, & \bar{k} \times \bar{i}=\widehat{n} \\
\overline{\bar{i}} \times \hat{l}=\bar{j}, & \overline{\bar{j}} \times \widehat{m}=\bar{k}, & \overline{\bar{k}} \times \widehat{n}=\bar{i}
\end{array}
$$

but for basis vectors

$$
\begin{gathered}
\frac{1}{\bar{i}}=\bar{i}, \quad \frac{1}{\bar{j}}=\bar{j}, \quad \frac{1}{\bar{k}}=\bar{k} \\
\bar{i} \times \hat{l}=\bar{j}, \quad \bar{j} \times \hat{m}=\bar{k}, \quad \bar{k} \times \widehat{n}=\bar{i}
\end{gathered}
$$

In cross product of the basis vectors one can notice the rule of sign selection of resulting vector. If the angular and rectilinear vectors are "equally" directed, the resulting rectilinear vector is positive, and vice versa. Then

$$
\bar{j} \times \hat{l}=-\bar{i}, \quad \bar{k} \times \hat{m}=-\bar{j}, \quad \bar{i} \times \hat{n}=-\bar{k}
$$

Let recall that a sequence of vectors in the cross product of vectors depends on the selected coordinate system.

Example 9: Let us find the force of the torque in coordinate-vector form.

$$
F=\frac{1}{h} M \quad-\text { is the general view of the equation to find the force of the torque }
$$

For this equation (for the right coordinate system with a positive angular direction counter-clockwise) the basis vectors have the following connections:

$$
\begin{gathered}
\bar{i} \times \hat{l}=\bar{j}, \quad \bar{j} \times \widehat{m}=\bar{k}, \quad \bar{k} \times \hat{n}=\bar{i}, \quad \bar{j} \times \hat{l}=-\bar{i}, \quad \bar{k} \times \widehat{m}=-\bar{j}, \quad \bar{i} \times \hat{n}=-\bar{k}, \quad \text { then } \\
\bar{F}=\frac{1}{\bar{h}} \times \widehat{M}=\overline{h^{\prime}} \times \widehat{M}=\left(h_{x}^{\prime} \bar{i}+h_{y}^{\prime} \bar{j}+h_{z}^{\prime} \bar{k}\right) \times\left(M_{x y} \widehat{l}+M_{y z} \hat{m}+M_{z x} \widehat{n}\right)= \\
=h_{x}^{\prime} M_{x y}(\bar{i} \times \widehat{l})+h_{x}^{\prime} M_{y z}(\bar{i} \times \widehat{m})+h_{x}^{\prime} M_{z x}(\bar{i} \times \widehat{n})+h_{y}^{\prime} M_{x y}(\bar{j} \times \widehat{l})+h_{y}^{\prime} M_{y z}(\bar{j} \times \widehat{m})+h_{y}^{\prime} M_{z x}(\bar{j} \times \widehat{n})+h_{z}^{\prime} M_{x y}(\bar{k} \times \widehat{l})+h_{z}^{\prime} M_{y z}(\bar{k} \times \widehat{m})+h_{z}^{\prime} M_{z x}(\bar{k} \times \widehat{n})= \\
=h_{x}^{\prime} M_{x y} \bar{j}+0-h_{x}^{\prime} M_{z x} \bar{k}-h_{y}^{\prime} M_{x y} \bar{i}+h_{y}^{\prime} M_{y z} \bar{k}+0+0-h_{z}^{\prime} M_{y z} \bar{j}+h_{z}^{\prime} M_{z x} \bar{i}= \\
=\left(h_{z}^{\prime} M_{z x}-h_{y}^{\prime} M_{x y}\right) \bar{i}+\left(h_{x}^{\prime} M_{x y}-h_{z}^{\prime} M_{y z}\right) \bar{j}+\left(h_{y}^{\prime} M_{y z}-h_{x}^{\prime} M_{z x}\right) \bar{k}= \\
=\left(\frac{h_{z} M_{z x}}{h_{x}^{2}+h_{y}^{2}+h_{z}^{2}}-\frac{h_{y} M_{x y}}{h_{x}^{2}+h_{y}^{2}+h_{z}^{2}}\right) \bar{i}+\left(\frac{h_{x} M_{x y}}{h_{x}^{2}+h_{y}^{2}+h_{z}^{2}}-\frac{h_{z} M_{y z}}{h_{x}^{2}+h_{y}^{2}+h_{z}^{2}}\right) \bar{j}+\left(\frac{h_{y} M_{y z}}{h_{x}^{2}+h_{y}^{2}+h_{z}^{2}}-\frac{h_{x} M_{z x}}{h_{x}^{2}+h_{y}^{2}+h_{z}^{2}}\right) \bar{k}= \\
=\left(\frac{h_{z} M_{z x}-h_{y} M_{x y}}{h_{x}^{2}+h_{y}^{2}+h_{z}^{2}}\right) \bar{i}+\left(\frac{h_{x} M_{x y}-h_{z} M_{y z}}{h_{x}^{2}+h_{y}^{2}+h_{z}^{2}}\right) \bar{j}+\left(\frac{h_{y} M_{y z}-h_{x} M_{z x}}{h_{x}^{2}+h_{y}^{2}+h_{z}^{2}}\right) \bar{k}=F_{x} \bar{i}+F_{y} \bar{j}+F_{z} \bar{k} \\
F_{x}=\frac{h_{z} M_{z x}-h_{y} M_{x y}}{h_{x}^{2}+h_{y}^{2}+h_{z}^{2}}, \quad F_{y}=\frac{h_{x} M_{x y}-h_{z} M_{y z}}{h_{x}^{2}+h_{y}^{2}+h_{z}^{2}}, \quad F_{z}=\frac{h_{y} M_{y z}-h_{x} M_{z x}}{h_{x}^{2}+h_{y}^{2}+h_{z}^{2}}
\end{gathered}
$$

Example 10: (Drogn \& Dubinin, 2005) 
Let us find tangential velocity in the coordinate-vector form.

$$
v=r \cdot w \quad-\text { the general form of the equation for tangential velocity solution }
$$

The basis vectors for this equation (for right-handed coordinate system with positive counter clockwise angular direction) have the following relation:

$$
\begin{aligned}
& \bar{i} \times \hat{l}=\bar{j}, \quad \bar{j} \times \hat{m}=\bar{k}, \quad \bar{k} \times \hat{n}=\bar{i}, \quad \bar{j} \times \hat{l}=-\bar{i}, \quad \bar{k} \times \hat{m}=-\bar{j}, \quad \bar{i} \times \hat{n}=-\bar{k}, \quad \text { then } \\
& \bar{v}=\bar{r} \times \widehat{w}=\left(r_{x} \bar{i}+r_{y} \bar{j}+r_{z} \bar{k}\right) \times\left(w_{x y} \hat{l}+w_{y z} \hat{m}+w_{z x} \widehat{n}\right)= \\
& =r_{x} w_{x y}(\bar{i} \times \hat{l})+r_{x} w_{y z}(\bar{i} \times \widehat{m})+r_{x} w_{z x}(\bar{i} \times \widehat{n})+r_{y} w_{x y}(\bar{j} \times \hat{l})+r_{y} w_{y z}(\bar{j} \times \widehat{m})+r_{y} w_{z x}(\bar{j} \times \widehat{n})+r_{z} w_{x y}(\bar{k} \times \hat{l})+r_{z} w_{y z}(\bar{k} \times \widehat{m})+r_{z} w_{z x}(\bar{k} \times \widehat{n})= \\
& =r_{x} w_{x y} \bar{j}+0-r_{x} w_{z x} \bar{k}-r_{y} w_{x y} \bar{i}+r_{y} w_{y z} \bar{k}+0+0-r_{z} w_{y z} \bar{j}+r_{z} w_{z x} \bar{i}= \\
& =\left(r_{z} w_{z x}-r_{y} w_{x y}\right) \bar{i}+\left(r_{x} w_{x y}-r_{z} w_{y z}\right) \bar{j}+\left(r_{y} w_{y z}-r_{x} w_{z x}\right) \bar{k}=v_{x} \bar{i}+v_{y} \bar{j}+v_{z} \bar{k} \\
& v_{x}=r_{z} w_{z x}-r_{y} w_{x y}, \quad v_{y}=r_{x} w_{x y}-r_{z} w_{y z}, \quad v_{z}=r_{y} w_{y z}-r_{x} w_{z x}
\end{aligned}
$$

\section{Results}

The main objective of this work is to draw attention to the problem of representations of mathematical models of vectors of angular quantities in the coordinate system and their inconsistency with physical quantities. Described theory of angular vectors solves this problem, we get matching directions of mathematical models with physical angular quantities. This made it possible to deduce equations in coordinate form for angular quantities that indicate their direction in the coordinate system.

Operations on angular vectors are similar to the operations on rectilinear vectors. This makes it easier to understand the general theory of vectors despite theory enlargement.

In examples 3 and 4 proved the miscarriage of the generally accepted statement that the result of the cross product of two rectilinear vectors is the rectilinear vector.

Examples 5, 6, 7 prove that the result of the cross product of two rectilinear vectors is the angular vector. Lets recite the main arguments of this statement:

Argument 1 (proof by contradiction). Of all possible cross products of vectors may exist only the described above. Examples 3, 4, 8 excludes the possibility of the existence of other cross products.

Argument 2. Since found by different methods the total result (angular vector) is the same (example 5) this vectors product exist.

Argument 3. Only this cross product observed in real life (examples 6 and 7).

Argument 4 . The possibility of the existence of the other cross products is excluding by a verbal proof in the general conclusion of a cross product.

Above described definition of the inversed vector and its properties (both rectilinear and angular). This vector allows us to perform vector cross division operations. As a result, it became possible to conclude in coordinate vector form formulas of physical quantities. As shown in examples 7 and 9 these formulas previously could not be obtained.

The operations of the scalar product of mixed vectors and the cross product of mixed vectors are considered.

The existence of a large number of examples demonstrates the applicability of the angular vector model and its agreement with the general theory of vectors.

The theory of vectors is the fundamental mathematical model, and these changes will improve the methodology of presenting of many physical regularities, and address a number of challenges.

Addendum: It is worth paying attention to one more problem. It is about the conventionally accepted positive left handed angular direction. This rule complicates the logical understanding of tasks, because people are accustomed to, that a positive angular direction is the direction of motion in a clockwise direction. This stereotype people remember from childhood. Moreover the task of any science is to facilitate understanding of a natural phenomenon, and I think you have to reckon with this stereotype. Another argument against the left-hand angular direction, is that this rule is accepted conditionally, without any logical explanation.

The best way to solve this problem is to change the name of the axes of coordinate system and basis vectors, fig.11. Then we don't need to display many of the formulas in the coordinate-vector form. 


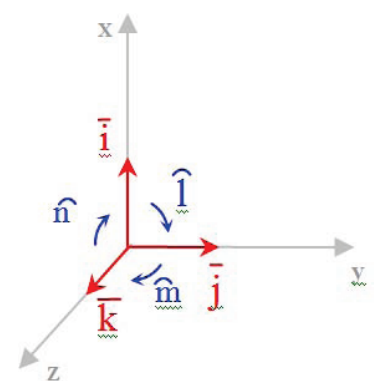

Fig. 11. Change of locations of coordinate axes and basis vectors

\section{Acknowledgements}

I would like to express my deep gratitude to Vishnevsky Maxim Viktorovich: to the first my reviewer and my old friend, mathematician and space industry specialist.

\section{References}

Borisenko, A. I., \& Tarapov, I. E. (1979). Vector and tensor analysis with applications. New York: Dover.

Boothroyd, G., \& Poli, C. (1980). Applied engineering mechanics.University of Massachusetts, NY:Marcel Deccer.

Dennis, G. Z., \& Michael, R. C. (2006). Advenced engineering mathematics. Jones \& Bartlett Learning.

Doran, ., \& Lasenby, . (2007). Geometric Algebra for Physicists. Cambridge University Press.

Drogn, V. I. \& Dubinin, V. V. (2005). Course of theoretical mechanics. Moscow: MSTU.

Khan, R. M. (2013). Analytical Geometry and Vector Algebra. Delhi, DEL, India:New Central Book Agency.

Khiman, A. D. (2015). Engineering mechanics. Statics and dynamics.

Rutherford, D. E. (2004). Vector Methods Applied to Differential Geometry, Mechanics, and Potential Theory. Mineola, New York: Dover.

Szolga, V. (2010). Theoretical mechanics, lecture notes and sample problems

Vasishtha, A. R. (2010). Vector algebra. Meerut,India: Krishna Prakashan.

Wilson, E. B. (1901). Vector Analysis: A text-book for the use of students of mathematics and physics, founded upon the lectures of J. Willard Gibbs. Yale University Press.

\section{Copyrights}

Copyright for this article is retained by the author(s), with first publication rights granted to the journal.

This is an open-access article distributed under the terms and conditions of the Creative Commons Attribution license (http://creativecommons.org/licenses/by/4.0/). 